\title{
Social Determinants of Health Themed Issue
}

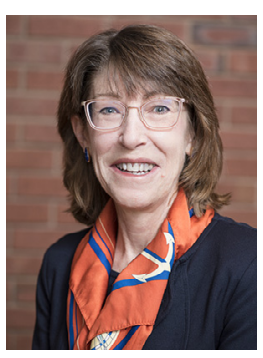

I hope you enjoy reading this issue of JNEB primarily focused on the social determinants of health (SDOH). The SDOH can be grouped into the 5 domains of economic stability, education access and quality, health care access and quality, neighborhood and built environment, and social and community context. $^{1}$

I wanted to also share some resources that may be of interest related to SDOH. One is the PhenX Toolkit that provides some standard data collection tools as recommended by the National Institute on Minority Health and Health Disparities. ${ }^{2}$ There are 16 protocols related to how to collect demographic data, including the preferred language for asking gender identity. There are 15 protocols relating to nutrition, most from our Nutrition Monitoring Programs, that I am sure everyone is familiar with. However, there are also some on parenting style, quality of the child's home environment, school social environment, and coping styles, which could be very useful in different areas of our research and practice.

The National Academies of Sciences, Engineering, and Medicine has developed a number of consensus reports, including Communities in
Action: Pathways to Health Equity. ${ }^{3}$ Not only does it cover the state of health inequity in the US, but also explores the causes of health inequity and the role of communities in promoting health equity, as well as tools used to promote health equity.

The SDOH literature summaries developed by the Department of Health and Human Services are not up to date. ${ }^{4}$ I was disappointed to not see JNEB in the food insecurity collection. However, if you search www. jneb.org for food insecurity you will find more than 300 research articles. Searching for health equity turns up almost 80 research articles. So, whether we are listed in larger federal resources, we are still here for your research and practice needs.

Our next themed issue and call for papers is on Virtual Learning, Telenutrition and eHealth Interventions, and the topic closes next month (March 2022). If your research is not yet complete, remember that JNEB accepts Great Educational Materials (GEMs), Perspectives, Reports, reviews of New Resources, and Research Methods related to both Interventions and Questionnaire Development. If you are unsure if your paper is a good fit, feel free to emailme at editor@jneb.org.

Finally, since it has been a month since JNEB went online only, I'd like your comments on the website, any accessibility issues, and suggestions for the future.

\author{
Karen Chapman-Novakofski, \\ PhD, RDN \\ Editor-in-Chief \\ Journal of Nutrition Education and \\ Behavior \\ Advancing Research, Practice, \\ and Policy
}

\section{REFERENCES}

1. Healthy People 2030. Social determinants of health. US Department of Health and Human Services, Office of Disease Prevention and Health Promotion. https://health.gov/healthypeople/ objectives-and-data/social-determinants-health. Accessed December 20, 2021.

2. National Institute on Minority Health and Health Disparities. PhenX Social Determinants of Health Toolkit. https:// www.nimhd.nih.gov/programs/collab/ phenx/index.html. Accessed December 21, 2021.

3. National Academies of Sciences, Engineering, and Medicine. In: Weinstein JN, Geller A, Negussie Y, Baciu A, eds. Communities in Action: Pathways to Health Equity. National Academies Press; 2017.

4. US Department of Health and Human Services. Social determinants of health literature summaries. https://health. gov/healthypeople/objectives-and-data/ social-determinants-health/literaturesummaries. Accessed December 21, 2021. 